\title{
Asymmetric extractions in orthodontics
}

\author{
Camilo Aquino Melgaço', Mônica Tirre de Souza Araújo²
}

Introduction: Extraction decisions are extremely important in during treatment planning. In addition to the extraction decision orthodontists have to choose what tooth should be extracted for the best solution of the problem and the esthetic/functional benefit of the patient.

Objective: This article aims at reviewing the literature relating the advantages, disadvantages and clinical implications of asymmetric extractions to orthodontics.

Methods: Keywords were selected in English and Portuguese and the EndNote 9 program was used for data base search in PubMed, Web of Science (WSc) and LILACS. The selected articles were case reports, original articles and prospective or retrospective case-control studies concerning asymmetrical extractions of permanent teeth for the treatment of malocclusions.

Conclusion: According to the literature reviewed asymmetric extractions can make some specific treatment mechanics easier. Cases finished with first permanent molars in Class II or III relationship in one or both sides seem not to cause esthetic or functional problems. However, diagnosis knowledge and mechanics control are essential for treatment success.

Keywords: Asymmetric extraction. Mechanics. Treatment. Orthodontics.

${ }^{1}$ MSc and PhD Student in Orthodontics, Federal University of Rio de Janeiro.

${ }^{2} \mathrm{MSc}$ and PhD in Orthodontics, UFRJ. Associate Professor of Orthodontics, Department of Pedodontics and Orthodontics, Federal University of Rio de Janeiro.

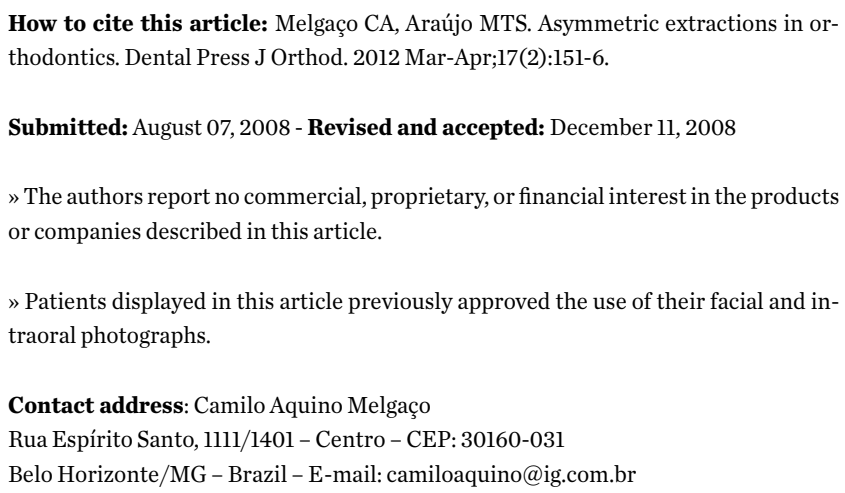

How to cite this article: Melgaço CA, Araújo MTS. Asymmetric extractions in orthodontics. Dental Press J Orthod. 2012 Mar-Apr;17(2):151-6.

Submitted: August 07, 2008 - Revised and accepted: December 11, 2008

» The authors report no commercial, proprietary, or financial interest in the products or companies described in this article.

" Patients displayed in this article previously approved the use of their facial and intraoral photographs.

Contact address: Camilo Aquino Melgaço

Rua Espírito Santo, 1111/1401 - Centro - CEP: 30160-031

Belo Horizonte/MG - Brazil - E-mail: camiloaquino@ig.com.br 


\section{INTRODUCTION}

The main goal of orthodontic treatment is to obtain a normal relationship of the teeth with facial structures. Edward H. Angle emphasized that the preservation of all dental units was necessary to achieve facial balance, harmony and esthetics. However, subsequent studies related to the stability of treatments pointed the necessity of teeth extractions in order to correct certain types of malocclusions. The lower incisor inclination revealed a great importance in diagnosis and orthodontic treatment planning. The orthodontists realized that maintaining all dental units in order to correct some kinds of malocclusions was not always possible. ${ }^{9,25,26,29}$ Besides, space closure due to extractions should be under total control of the professional. In cases of dental position asymmetries, this control would ensure functional and adequate occlusion of the teeth at the end of treatment. ${ }^{3,18}$

Therefore, many authors have suggested that asymmetric extractions would be necessary and important to correct midline deviation, favoring unilateral movement of the posterior teeth, reduce treatment time and tooth movement, facilitate orthodontic mechanics and obtain more stable and functional results. ${ }^{6,8,15,18,23,30}$ This study aims to conduct a literature review about asymmetric extractions, emphasizing the importance of this procedure for orthodontics.

\section{MATERIALS AND METHODS}

Selected keywords in English (asymmetric, extraction, treatment, orthodontics, mechanics) and Portuguese (extrações assimétricas, tratamento, ortodontia and mecânica) were used in EndNote 9 software, searching for studies in the following databases: PubMed, Web of Science (WSC) and LILACS. These keywords should be present in one of the following parts of the articles: Title, keywords, abstract or text. Following, inclusion / exclusion criteria were determined in order to select the papers. Studies reporting teeth agenesis or pathologies, systemic diseases, syndromes, cleft palate, orthognathic surgery and conventional extractions of first premolars were excluded. Papers including case reports, original articles, case-control studies involving prospective or retrospective mechanical or asymmetric extractions of permanent teeth were selected. A total of 30 articles were chosen for the literature review and discussion.

\section{LITERATURE REVIEW AND DISCUSSION}

The perfect symmetry is a theoretical concept and rarely found in nature. The facial and dental asymmetry is a common phenomenon often observed when comparing right and left sides. ${ }^{1,25}$ According to Lundström, ${ }^{19}$ the asymmetries of the arches could be classified as quantitative or qualitative. Imbalanced numbers of teeth on each side of the arch would be included in the quantitative asymmetries. Differences in teeth size and position, associated to dental arch relationships to the skull and to themselves would be grouped in qualitative asymmetries. The treatment of quantitative asymmetries, for example, could be accomplished by prosthetic rehabilitation or fixed orthodontics. ${ }^{1}$ Stripping, single incisor extraction and asymmetric extraction of permanent teeth followed by asymmetrical mechanics would be good options for treating qualitative asymmetries. ${ }^{1,2,6,8,16,30}$

Garn, Lewis and Kerewsky ${ }^{7}$ observed that asymmetries in tooth size generally did not involve an entire side of the arch. Teeth of the same morphological class tend to have the same asymmetry. If the first right premolar is larger than the left, the same trend could be expected between the second premolars. Melgaço et al ${ }^{20}$ measured the mesiodistal widths of all permanent teeth (excepted $3^{\text {rd }}$ molars) of 500 dental casts and found no statistically significant differences between right and left sides. Other authors also found similar results. ${ }^{5,17}$

Therefore, in cases of qualitative or quantitative asymmetries, the extraction of teeth should be considered as a treatment option to achieve facial balance and occlusion stability at the end of the treatment. However, extracting teeth is still a challenge for orthodontists, especially in choosing which tooth should be extracted. ${ }^{16}$ In these cases, Rheude et $\mathrm{al}^{22}$ emphasizes the importance of the plaster casts for orthodontic treatment planning in order to perform asymmetric extractions.

Thus, the Bolton analysis determines the ratio of the mesiodistal widths of maxillary versus mandibular teeth. ${ }^{2}$ This tooth-size discrepancy allows a 

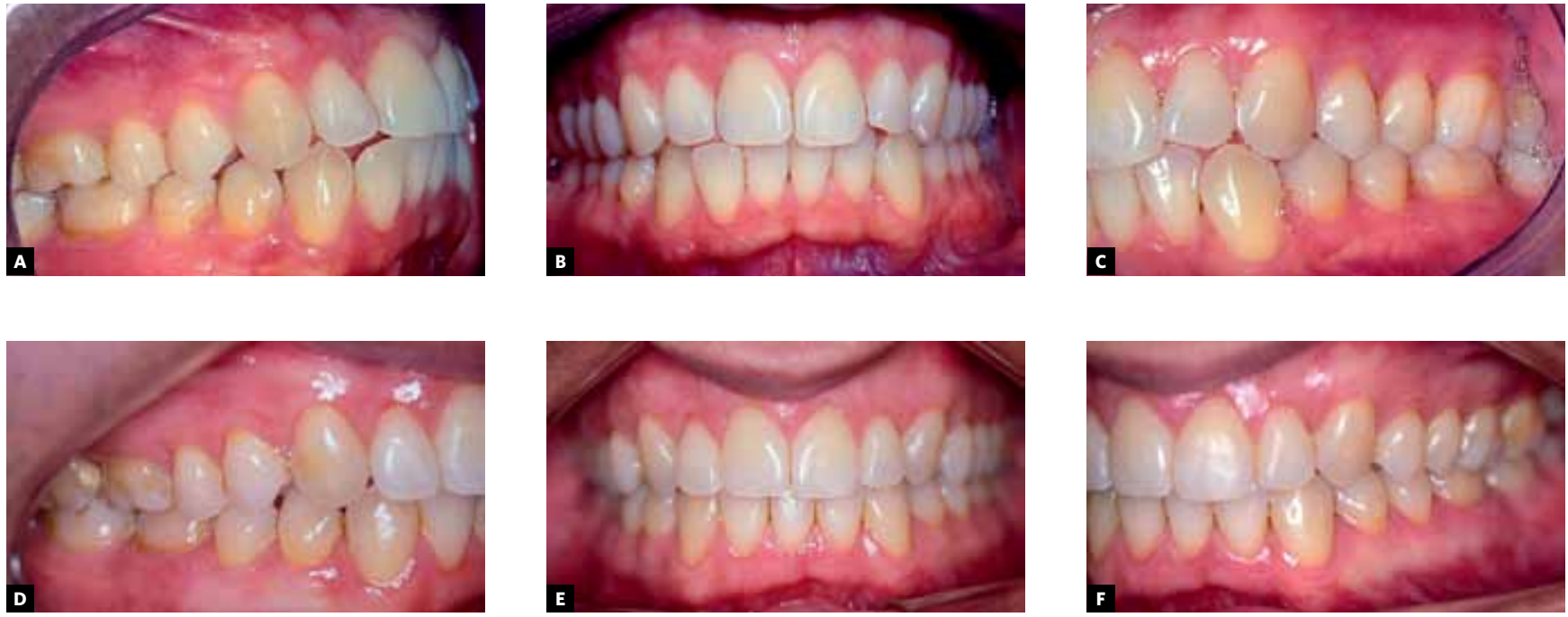

Figure 1 - Patient presenting a Bolton discrepancy with anterior excess. Treatment performed with extraction of only a lower permanent incisor.

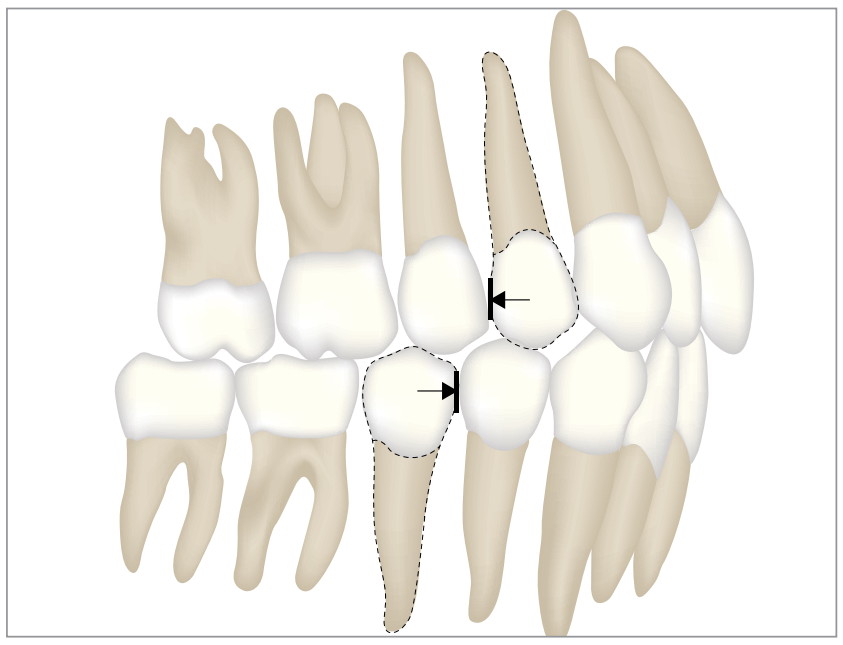

Figure 2 - Asymmetric extractions: First premolars and second premolars.

further option of extraction: Removal of one lower incisor. According to Klein ${ }^{16}$ this option can provide satisfactory results in some specific cases, as observed in Figure 1.

In Class II malocclusions cases with small lower anterior crowding or slight protrusion of the lower incisors, the extraction of lower second premolars and upper first premolars is a good treatment option (Fig 2). This favors the correction of the protruding upper anterior teeth (if present) and favors the correction of lower anterior crowding or some slight protrusion of the lower incisors, without great facial impact. The correction of molar relationship would be achieved by more anchorage loss in the mandible. The use of Class II elastics would facilitate this process, associated with a face-bow or a transpalatal arch to avoid maxillary anchorage loss. However, patient's collaboration would be essential for the success of this kind of therapy. ${ }^{21,28.29}$ The removal of a first premolar on one side and the second premolar on the other can also occur, depending on arch discrepancy location. ${ }^{28,29}$

The most discussed and common cases about asymmetric extractions refer to Class II subdivision malocclusions with lower dental midline deviation. The solution in these situations arises from symmetrical extraction of four first premolars or asymmetric extractions of only three premolars. However, the first choice requires greater collaboration of the patient, since the use of intermaxillary elastics become necessary. On the other hand, extraction of two upper premolars and only one lower premolar (opposite to the midline deviation) results in asymmetrical molar relationship at the end of the treatment (Class I and Class II). ${ }^{10-13,21,27}$ Thus, in these situations it would be easier to achieve midline correction and obtain better vertical control. Treatment considering three premolars extraction protocol in Class II subdivision cases requires less treatment time, providing more satisfactory results. ${ }^{10,12}$ Janson et al ${ }^{10}$ also state that the asymmetric extraction protocol is best suited for promoting minor changes in the profile. 
However, the authors described an extrusion of lower incisors in cases treated with three premolars extraction protocol and affirmed that better vertical control of the anterior teeth is accomplished when symmetrical extractions are performed. They also concluded that an asymmetry in the position of the first permanent molars is inevitable in these cases. This was also confirmed by Janson et $\mathrm{al}^{11}$ and Johnson and Smith. ${ }^{14}$ Other authors state that asymmetric extractions (first premolar on one side and second premolar on the other) could help to correct midline deviations, anterior crowding and reduce the protrusion of the lower incisors in Class II subdivision malocclusions. ${ }^{25,30}$ However, Gianelly et $\mathrm{al}^{8}$ reported that the extraction of four first premolars would have the advantage of using an easier mechanical anchorage control, and to preserve the contact point between the second premolar and first molar (more anatomically correct and preferable than the contact of the first premolar with the first molar).

Based on these assumptions, Burstone ${ }^{4}$ defined three types of space closure control in extraction sites:

» Type $\mathrm{A}$ - the teeth anterior to those extracted would occupy all or almost all of the extraction sites.

» Type B - space closure would be a result of both anterior retraction and posterior anchorage loss.

» Type $\mathrm{C}$ - the teeth posterior to those extracted would occupy all or almost all of the extraction sites.

These space closure controls must not be identical on both sides of the dental arch. In asymmetrical malocclusions, for example, it would be required a type $\mathrm{C}$ space closure on the right side and a type $\mathrm{A}$ space closure on the left side. These differences in mechanics would be essential to achieve treatment goals as midline correction, appropriate anteroposterior positioning of the teeth, improving facial profile and dental stability. ${ }^{3,18}$ As stated before, asymmetric extractions could result in asymmetrical position of the molars. The final molar relationship could be Class II or III only in one side or in both sides ${ }^{21,30}$ (Fig 3 ).

However, space closure in asymmetric extraction cases is difficult due to anchorage control. A gap in extraction sites could persist even after correction of midline, solving anterior crowding and improving profile. In these cases, molar mesialization should not change the final position of the lower incisors nor affect facial harmony. The closing loop bends or elastic ligatures could deviate the midline or compromise canine relationship. ${ }^{8,30}$ Fiorentino and Melsen ${ }^{6}$ agreed that space closure in the posterior region of the arch is a difficult procedure, especially in Class II malocclusions. Thus, Bishara, Burkey and Kharouf $^{1}$ suggest the use of unilateral Class II or Class III elastics, associated with oblique anterior elastics to produce asymmetrical forces. In order to have total control of the mechanics, the system and braces used must deliver the correct and precise forces needed to facilitate tooth movement, causing minimal tissue damage and discomfort to the patient. ${ }^{3,18}$

Asymmetric extractions could resolve cases that would require the use of prostheses or implants. ${ }^{6}$ Slavicek ${ }^{24}$ postulate the use of orthognathic surgery or asymmetric extractions of upper first premolars as an alternative treatment for severe Class II malocclusion cases with retruded mandible and no growth potential. In these cases, the mechanics should favor the retraction of maxillary anterior teeth and the final molar relationship would remain in Class II on both sides. However, normal canine relationships would be present (Fig 4). 

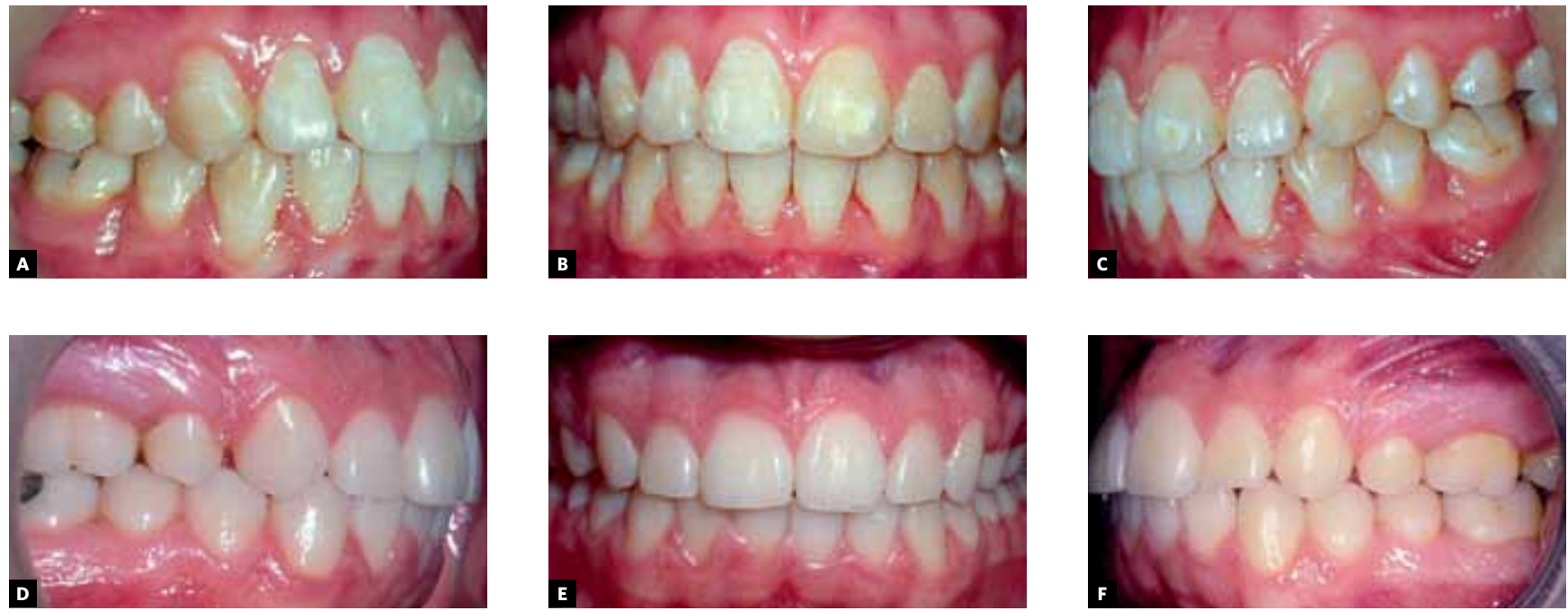

Figure 3 - A, B, C) Finalization with first permanent molars in Class III relationship. D, E, F) Finalization with first permanent molars in Class II relationship.

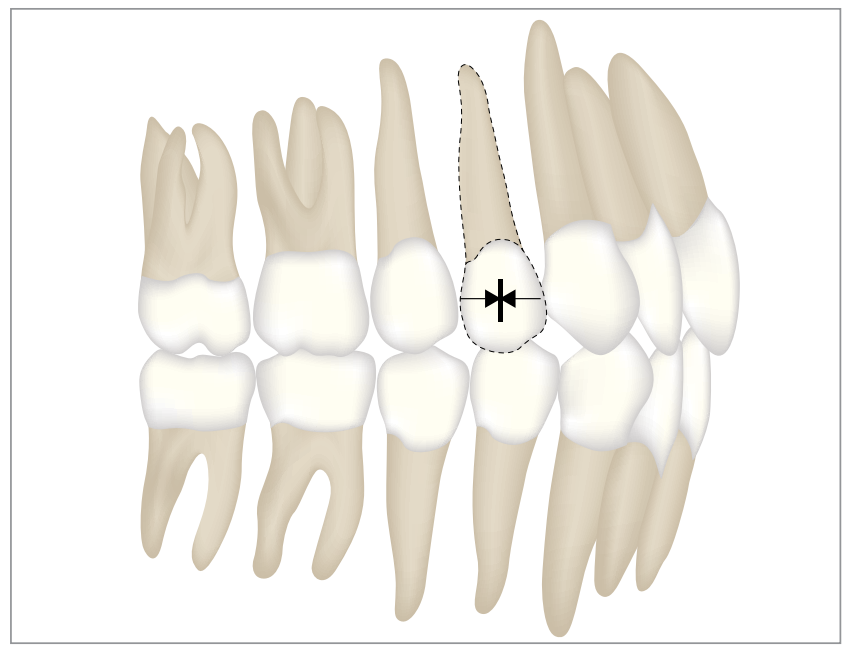

Figure 4 - Asymmetric extractions: only upper first premolars.

\section{FINAL CONSIDERATIONS}

According to the reviewed literature, asymmetric extractions could simplify and facilitate orthodontic treatment and mechanics in some specific cases. As a result, first molars relationship could differ for right or left sides and this asymmetry would not bring functional or esthetics problems. In cases of Bolton discrepancy, a lower incisor extraction option should be considered. However, the orthodontist must have total control of the mechanics used to achieve the best final results at the end of the treatment. 


\section{REFERENCES}

1. Bishara SE, Burkey PS, Kharouf JG. Dental and facial asymmetries: a review. Angle Orthod. 1994;64(2):89-98

2. Bolton WA. Disharmony in tooth size and its relation to the analysis and treatment of malocclusion. Angle Orthod. 1958;28(3):113-30

3. Braun S, Sjursen RC Jr, Legan HL. On the management of extraction sites. Am J Orthod Dentofacial Orthop. 1997;112(6):645-55.

4. Burstone CJ. The segmented arch approach to space closure. Am J Orthod. 1982;82(5):361-78.

5. Cecílio E, Vigorito JW. Avaliação do índice de Moyers na predição das dimensões mésio-distais de caninos e premolares em pacientes adolescentes, brasileiros, leucodermas, do sexo masculino e feminino. Ortodontia. 2001:34:8-15.

6. Fiorentino G, Melsen B. Case report: asymmetric mandibular space closure. J Clin Orthod. 1996;30(9):519-23

7. Garn $S M$, Lewis $A B$, Kerewsky RS. The meaning of bilateral asymmetry in the permanent dentition. Angle Orthod. 1996;36(1):55-62.

8. Gianelly AA, Smith JB, Bednar JR, Dietz VS. Asymmetric space closure. Am J Orthod. 1986;90(4):335-41.

9. Hulsey CM. An esthetic evaluation of lip-teeth relationships present in the smile. Am J Orthod. 1970;57(2):132-44.

10. Janson G, Carvalho PEG, Cançado RH, Freitas MR, Henriques JFC. Cephalometric evaluation of symmetric and asymmetric extraction treatment for patients with Class II subdivision malocclusions. Am J Orthod Dentofacial Orthop. 2007;132(1):28-35.

11. Janson G, Cruz KS, Woodside DG, Metaxas A, Freitas MR, Henriques JF. Dentoskeletal treatment changes in Class II subdivision malocclusions in submentovertex and posteroanterior radiographs. Am J Orthod Dentofacial Orthop. 2004;126(4):451-63.

12. Janson GRP, Dainesi EA, Henriques JFC, Freitas MR, Lima KJRS. Class II subdivision treatment success rate with symmetric and asymmetric extraction protocols. Am J Orthod Dentofacial Orthop. 2003;124(3):257-64.

13. Janson GRP, Metaxas A, Woodside DG, Freitas MR, Pinzan AP. Three-dimensional evaluation of skeletal and dental asymmetries in Class II subdivision malocclusions. Am J Orthod Dentofacial Orthop. 2001;119(4):406-18.

14. Johnson DK, Smith RJ. Smile esthetics after orthodontic treatment with and without extraction of four first premolars. Am J Orthod. 1995;108(2):162-7.
15. Jones O, Ortiz C, Schein R. Orthodontic management of a patient with Class I malocclusion and severe crowding. Am J Orthod. 1990;98(3):189-96.

16. Klein DJ. The mandibular central incisor, an extraction option. Am J Orthod 1997;111(3):192-9.

17. Legovic M, Novosel A, Legovic A. Regression equations for determining mesiodistal crown diameters of canines and premolars. Angle Orthod. 2003;73(3):314-8.

18. Lima MH, Oliveira JHG, Tanaka O. Extrações assimétricas de dentes permanentes na correção da linha mediana. J Bras Ortodon Ortop Facial. 2002;7(37):78-86.

19. Lundstron A. Some asymmetries of the dental arches, jaws, and skull, and their etiological significance. Am J Orthod. 1961;47(2):81-106.

20. Melgaço CA, Araújo MTS, Ruellas ACO. Lower first permanent molars and incisors as predictors of lower permanent canine and premolar widths. Am J Orthod Dentofacial Orthop. 2007;132(3):340-5

21. Rebellato J. Asymmetric extractions used in the treatment of patients with asymmetries. Semin Orthod. 1998;4:180-8.

22. Rheude B, Sadowsky PL, Ferriera A, Jacobson A. An evaluation of the use of digital study models in orthodontic diagnosis and treatment planning. Angle Orthod. 2005;75(3):300-4.

23. Shroff B, Siegel SM. Treatment of patients with asymmetries using asymmetric mechanics. Semin Orthod. 1998;4(3):165-79.

24. Slavicek R. Clinical and instrumental functional analysis for diagnosis and treatment planning. Part 2. J Clin Orthod. 1988;22(7):430-43.

25. Tayer BH. The asymmetric extraction decision. Angle Orthod. 1992;62(4):291-7.

26. Tweed CH. A philosophy of orthodontic treatment. Am J Orthod. 1945;31(2):74-113.

27. Todd M, Hosier M, Sheehan T, Kinser D. Asymmetric extraction treatment of a Class II Division 1 subdivision left malocclusion with anterior and posterior crossbites. Am J Orthod Dentofacial Orthop. 1999;115(4):410-7.

28. Vaden J, Dale JG, Klontz HA. Tweed-Merrifield edgewise appliance: philosophy, diagnosis and treatment. In: Graber TM, Vanarsdall RL Jr. Orthodontics. Current principles and techniques. $3^{\text {rd }}$ ed. St. Louis: Mosby; 2000. p. 647-707.

29. Vaden JL, Kiser HE. Straight talk about extraction and nonextraction: a differential diagnostic decision. Am J Orthod. 1996;109(4):445-52.

30. Whitley JB. A Class II, Division 1 malocclusion: a malocclusion with a significant mandibular arch length deficiency. Am J Orthod. 1996;110(6):688-93. 